\title{
Optimization of Arrays of Gold Nanodisks for Plasmon-Mediated Brillouin Light Scattering
}

\author{
W. L. Johnson* and S. A. Kim \\ Materials Reliability Division, National Institute of Standards and Technology, 325 Broadway, \\ Boulder, Colorado 80305 \\ Z. N. Utegulov ${ }^{\dagger}$ \\ Department of Chemistry, University of Nebraska-Lincoln, 520 Hamilton Hall, Lincoln, Nebraska 68588
}

\begin{abstract}
J. M. Shaw
Electromagnetics Division, National Institute of Standards and Technology, 325 Broadway, Boulder, Colorado 80305
\end{abstract}

\author{
B. T. Draine \\ Department of Astrophysical Sciences, Princeton University, 108 Peyton Hall, Princeton, New Jersey 08544
}

Received: April 29, 2009; Revised Manuscript Received: June 26, 2009

\begin{abstract}
Distributions of electric fields in two-dimensional arrays of gold nanodisks on a $\mathrm{Si}_{3} \mathrm{~N}_{4}$ membrane, with light incident through the membrane, are modeled with the aim of determining array geometries for effective plasmon-mediated Brillouin light scattering ("surface-enhanced Brillouin scattering") from phonons or magnons in a specimen placed in contact with such an array. Particular attention is devoted to average intensities and higher-wavevector components of the fields in a plane $2 \mathrm{~nm}$ from the circular nanodisk/vacuum interface, which is anticipated to be in the vicinity of the surface of a specimen. For nanodisks with diameters of 50 $\mathrm{nm}$, the average intensity near the circular nanodisk/vacuum interface increases as the angle of the incident light approaches the normal of the $\mathrm{Si}_{3} \mathrm{~N}_{4}$ surface. At low angles of incidence relative to the $\mathrm{Si}_{3} \mathrm{~N}_{4}$ normal, average intensities also increase with decreasing array spacing, primarily because of the corresponding changes in fractional coverage area of the gold. The highest average intensities (with near-normal incidence and 70 $\mathrm{nm}$ array periodicity) are found to be $\sim 3$ times that of the incident light. More significantly, higher-wavevector components of the fields are found to have intensities comparable to the incident light. This finding provides evidence for the feasibility of using surface plasmons in nanodisk or nanoline arrays to mediate Brillouin scattering from phonons or magnons with wavelengths of a few tens of nanometers, which would extend the wavevector range of Brillouin-scattering metrology by an order of magnitude.
\end{abstract}

\section{Introduction}

Resonant excitation of surface plasmons in noble-metal nanoparticles and nanodot arrays has been extensively employed in a number of optical spectroscopic metrologies to generate high-intensity localized fields and associated enhancement of spectral intensities. ${ }^{1,2}$ In surface-enhanced Raman spectroscopy (SERS), for example, surface-plasmon mediation of nanoparticles can lead to enhancement of spectral peak intensities of adhered molecules by many orders of magnitude.

One optical method for which surface-plasmon mediation has been found, thus far, to be less useful is Brillouin light scattering (BLS), which involves inelastic scattering of light from acoustic phonons or magnons in a manner analogous to the scattering from optical phonons in Raman spectroscopy. Attempts to implement plasmon-mediated BLS (or "surface-enhanced Brillouin scattering", SEBS) with randomly arranged silver nanoparticles on a surface, as has been done in SERS, have not yielded significant increases in spectral intensities. ${ }^{3}$ To understand the relative ineffectiveness of this approach, one should

* To whom correspondence should be addressed.

${ }^{\dagger}$ Present address: Idaho National Laboratory, P.O Box 1625, Idaho Falls, ID $83415-2211$. note that BLS from acoustic phonons is a spatially coherent scattering process (Bragg scattering) involving phonons with a wavevector specified by the scattering geometry, and such selectivity of the wavevector is necessary to obtain a useful spectrum because of the strong wavevector dependence of acoustic-phonon frequencies. As described by Moretti et al., ${ }^{3}$ the localization of fields in a random array of $\mathrm{Ag}$ nanoparticles corresponds to a broad range of wavevectors and, therefore, is not expected to contribute to well-defined spectra. In contrast, optical phonons detected in Raman spectroscopy have relatively flat dispersion curves over a significant fraction of the Brillouin zone, and therefore, well-defined peaks are obtained even with a broad distribution of wavevectors.

One effective approach to SEBS, first proposed by Fukui and co-workers, ${ }^{4,5}$ employs uniform noble-metal films with light incident through a Kretschmann hemispherical prism to provide overlap of the photon and extended-surface-plasmon dispersion curves. Several experimental studies ${ }^{3,6-8}$ have used this approach with Ag films and found intensities of spectral peaks from phonons in the Ag/glass to be increased by factors as high as $750 .{ }^{6}$ The Kretschmann geometry has also been used to enhance peak intensities from dielectric films deposited on a noble-metal film. Lee et al. ${ }^{9}$ used this approach to study Langmuir-Blodgett 
films of cadmium arachidate, and Eberle and Pietralla ${ }^{10}$ used it to study periodic arrays of polymeric nanolines. One limitation of the Kretschmann geometry is that it restricts practical measurements to a narrow range of phonon wavevectors (unless the specimen is a phononic crystal, as in the work of Eberle and Pietralla), ${ }^{10}$ since high field intensities in the $\mathrm{Ag}$ are achieved only at the critical angle for attenuated total reflection. The restriction of a narrow range of phonon wavevectors can be relaxed, and the need for a Kretschmann prism can be eliminated, by employing a periodically corrugated noble-metal film for plasmon mediation. ${ }^{11,12}$

In a previous report, ${ }^{13}$ we suggested that periodic arrays of noble-metal nanodisks or nanolines may provide a more flexible platform than the Kretschmann geometry or corrugated noblemetal films for SEBS, enabling measurements over a wider range of incident angles. Our measurements on gold-nanodisk arrays on glass provided evidence for modest enhancement (factors of 2-6) of phonon spectra from this structure over a relatively broad range of angles. We also suggested that, if specimens are placed in contact with such arrays, nanoscale variations of field intensities may enable an extension of the range of wavevectors detectable in BLS, since electric fields in a two-dimensional array have discrete wavevector components equal to the surface-parallel component of the wavevector of the incident light plus an integral multiple of the reciprocal lattice vector(s) of the array. Inelastic scattering of higherwavevector components of these fields is expected to occur from acoustic waves or spin waves with correspondingly high wavevectors, and the associated energy loss may be effectively translated back to far-field backscattered light through reversed mediation of surface plasmons, in a manner similar to the twoway surface-plasmon mediation of SERS. ${ }^{1}$

Robertson et al. ${ }^{11,12}$ found a similar role of reciprocal lattice vectors in the wavevector-conservation relation in SEBS measurements on corrugated continuous Ag films on a glass substrate. In that study, the detected phonons were in the Ag/ substrate, since no other specimen was placed in contact with the Ag. As described by Robertson et al., ${ }^{11,12}$ the usual wavevector-conservation relation for BLS from phonons in an opaque half space

$$
(\mathbf{q})_{\|}=\left(\mathbf{k}_{0}\right)_{\|}+\left(\mathbf{k}_{\mathrm{s}}\right)_{\|}
$$

is modified in the presence of a periodic surface grating, such that

$$
(\mathbf{q})_{\|}=\left(\mathbf{k}_{0}\right)_{\|}+\left(\mathbf{k}_{\mathrm{s}}\right)_{\|}+\mathbf{K}
$$

where $(\mathbf{q})_{\|}$is the surface-parallel component of a phonon wavevector, $\left(\mathbf{k}_{0}\right)_{\|}$and $\left(\mathbf{k}_{\mathrm{s}}\right)_{\|}$are, respectively, the components of the wavevectors of the incident and scattered light parallel to the surface, and $\mathbf{K}$ is a reciprocal lattice vector of the grating. Equation 2 is also valid more generally for BLS from any structure that is elastically periodic in a plane (any twodimensional phononic crystal). ${ }^{14-16}$ However, Robertson et al. ${ }^{12}$ showed that, in the case of corrugated Ag films, surface plasmons are intermediate states between photons and phonons. Their results, therefore, suggest that similar scattering involving reciprocal lattice vectors will occur if a specimen is placed in nanoscale contact with a noble-metal thin-film array.

In this report, we consider only the initial elastic part of the above scattering process, involving coupling of incident light to suface plasmons in nanodisk arrays. We present calculations

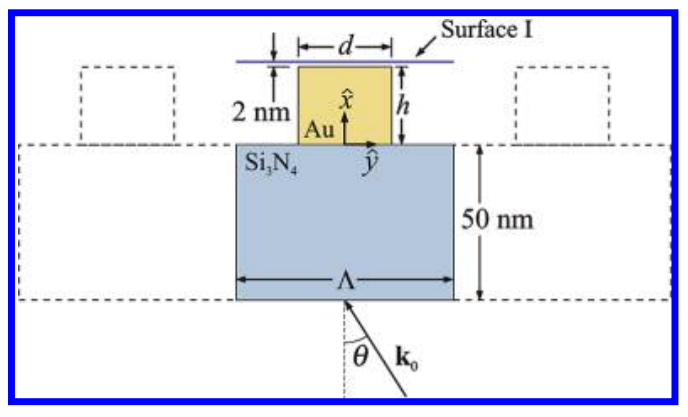

Figure 1. Cross-sectional geometry of the unit cell and definitions of axes and dimensional parameters. Dashed lines indicate adjacent unit cells. The unit vector $\hat{z}$ is into the page, and the origin $(x=y=z=$ 0 ) of the coordinate system is at the center of the bottom circular surface $\left(\mathrm{Au} / \mathrm{Si}_{3} \mathrm{~N}_{4}\right.$ interface) of one of the nanodisks.

of electric-field distributions as a function of geometric parameters of arrays with the aim of providing guidance in the design of arrays for SEBS and estimating the magnitude of higherwavevector components of the fields. Particular attention is paid to intensities in vacuum near the circular surface of the nanodisks, with light incident through the substrate of the array. This scattering geometry differs from that in most previous studies of surface plasmons in nanodot arrays, which have generally considered light incident directly on the metallic surfaces of the arrays, and is relevant also to potential applications of surface-plasmon enhancement in photovoltaics ${ }^{17-21}$ and nanolithography. ${ }^{22,23}$

Although a substantial number of papers have focused on the excitation of surface plasmons in arrays of noble-metal disks, spheres, or pyramids, ${ }^{22,24-38}$ only a small fraction of the extensive parameter space of such arrays (including target geometry, material properties, and incident wavevector) has been explored. Because complete field calculations are computationally intensive, most modeling studies have been restricted to normal-incidence far-field extinction, absorption, or scattering.

\section{Methods}

The specific targets considered in this study are twodimensionally periodic square arrays of gold nanodisks supported by $\mathrm{Si}_{3} \mathrm{~N}_{4}$ membranes. Membranes with nanoscale thickness are chosen as substrates because they provide volumes of the unit cells of the arrays that are small enough to enable practical computation times. These small volumes are also attractive with respect to future BLS measurements, since they will minimize the intensity of spectral peaks from the substrate. The particular choice of $\mathrm{Si}_{3} \mathrm{~N}_{4}$ arises from the practical consideration that membranes of this material are readily available commercially.

Our primary focus is on nanodisks with a diameter of 50 $\mathrm{nm}$. For this diameter, electric fields are calculated as a function of nanodisk height, nanodisk spacing, and angle of the wavevector of incident light.

The coordinate system, orientation of the incident wavevector, and dimensional parameters of a unit cell of the array are defined in Figure 1. The gold nanodisk is a solid cylinder with diameter $d$ and height $h$. The array is periodic in $y$ and $z$ with a spacing $\Lambda$ in both directions. The thickness of the $\mathrm{Si}_{3} \mathrm{~N}_{4}$ substrate is 50 $\mathrm{nm}$. Surface I is defined to be parallel to the surfaces of the substrate, to be $2 \mathrm{~nm}$ above the top circular surface of the gold, and to extend to the edges of the unit cell, such that its square area is $\Lambda \times \Lambda$. 


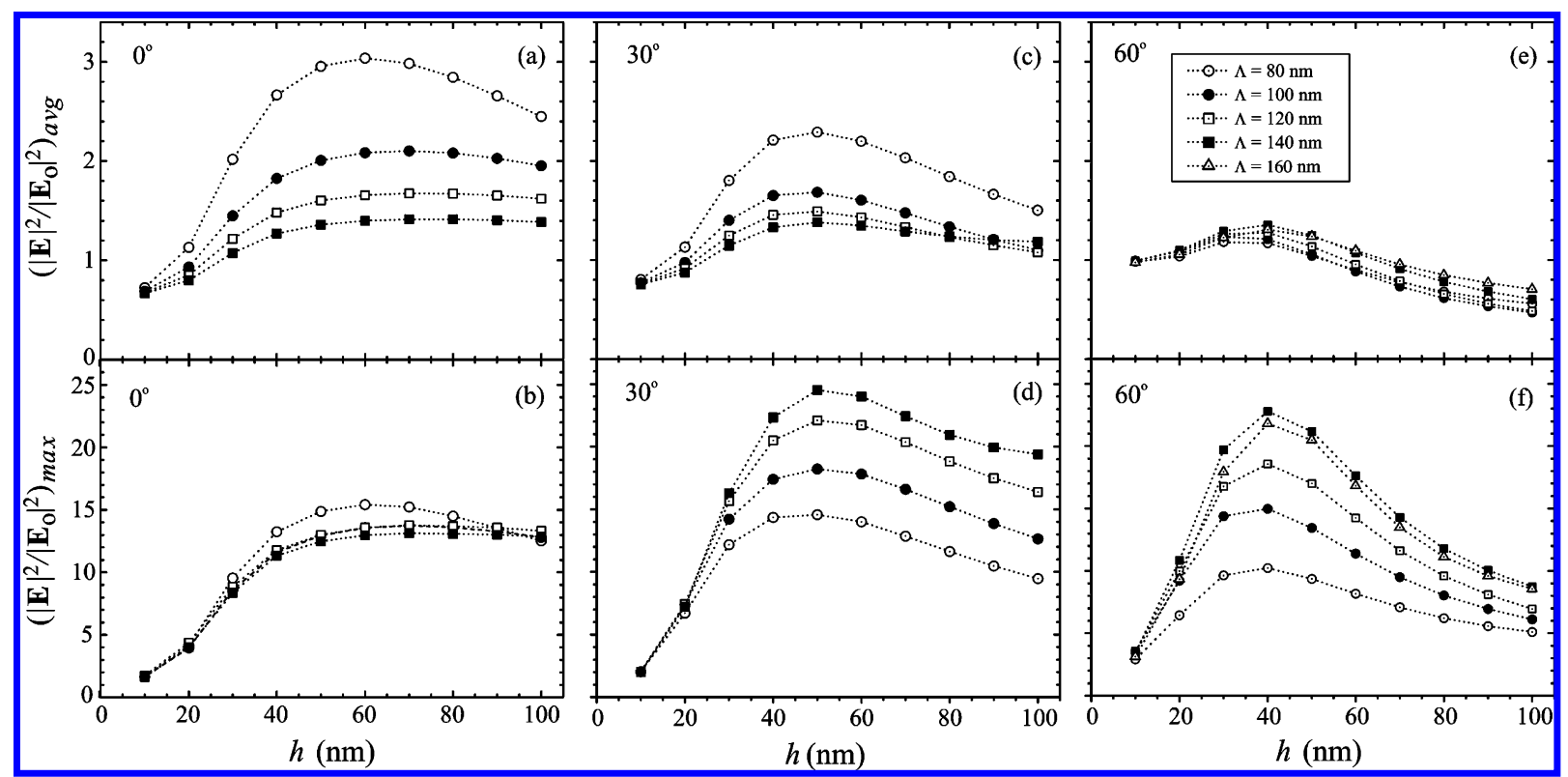

Figure 2. Average intensities $\left(|\mathbf{E}|^{2} /\left|\mathbf{E}_{0}\right|^{2}\right)_{\text {avg }}$ and maximum intensities $\left(|\mathbf{E}|^{2} /\left|\mathbf{E}_{0}\right|^{2}\right)_{\max }$ over surface I as a function of nanodisk height $h$ for $\theta=0^{\circ}($ a and b), $\theta=30^{\circ}$ (c and d), and $\theta=60^{\circ}$ (e and f). Spacing $\Lambda=80$ to $160 \mathrm{~nm}$. Diameter $d=50 \mathrm{~nm}$. DDSCAT dipole spacing $=5 \mathrm{~nm}$.

The electric field of the incident light is given by

$$
\mathbf{E}_{i n c}(\mathbf{r}, t)=\mathbf{E}_{0} \exp \left[i\left(\mathbf{k}_{0} \cdot \mathbf{r}-\omega t\right)\right]
$$

where $\mathbf{E}_{0}$ and the wavevector $\mathbf{k}_{0}$ lie in the $x-y$ plane. The wavelength $2 \pi /\left|\mathbf{k}_{0}\right|$ is chosen to be $532 \mathrm{~nm}$, except where otherwise noted (in absorption spectra), because this is a wavelength commonly employed in BLS systems.

We employ the discrete dipole approximation ${ }^{39-41}$ to calculate the electromagnetic fields in periodic arrays with unit cells having the geometry shown in Figure 1. The calculations were carried out with version 7.0.1 of the open-source code DDSCAT. ${ }^{42,43}$ As input to the calculations, the complex index of refraction of gold as a function of wavelength was taken to be that measured by Johnson and Christy ${ }^{44}$ for an evaporated gold film. The real part $n$ of the index of refraction of $\mathrm{Si}_{3} \mathrm{~N}_{4}$ was taken from the experimental results of Philipp. ${ }^{45}$ Since Philipp does not report values of the imaginary part $\kappa$ of the index of refraction of $\mathrm{Si}_{3} \mathrm{~N}_{4}$ in the range of wavelengths of interest here, values of $\kappa$ were taken from the modified Lorentzoscillator model of Djurišić and $\mathrm{Li}^{46}{ }^{46}$ which was fit to Philipp's ${ }^{45}$ data at shorter wavelengths. At $532 \mathrm{~nm}$, the indices of refraction were $n=0.544$ and $\kappa=2.231$ for gold and $n=2.036$ and $\kappa$ $=0.016$ for $\mathrm{Si}_{3} \mathrm{~N}_{4}$.

Surface-plasmon fields vary significantly on a single-nanometer scale. Therefore, discrete-dipole calculations ideally should be performed with a dipole-grid spacing of $\sim 1 \mathrm{~nm}$ or less. However, considering the relatively wide range of parameter space explored in this study, the computation time that would be involved in such calculations is prohibitive. Our calculations were performed with grid spacings of 2.5 or $5 \mathrm{~nm}$ (as indicated in figure captions, below). These spacings are sufficient for the purposes of this study, which are to determine the form and trends in the intensity distributions as a function of geometric parameters and to estimate the magnitude of higher-wavevector components of the fields.

\section{Results}

As described above, the electric-field intensities over surface I are expected to affect the magnitude of inelastic scattering from phonons or magnons in a specimen placed in contact with the nanodisk array. In this section, the values of average and maximum intensities over this surface are calculated as a function of nanodisk aspect ratio, spacing, and angle of incident light. Absorption spectra are also presented. Finally, the intensities of higher-wavevector components of the fields are determined through spatial Fourier analysis.

A. Aspect Ratio. Figure 2 shows calculations of normalized average intensity $\left(|\mathbf{E}|^{2} /\left|\mathbf{E}_{0}\right|^{2}\right)$ avg and normalized maximum intensity $\left(|\mathbf{E}|^{2} /\left|\mathbf{E}_{0}\right|^{2}\right)_{\max }$ over surface I as a function of $h$, where $\mathbf{E}$ is the total electric field (including the incident field and the field from the infinite set of discrete dipoles modeling the $\mathrm{Au}$ and $\mathrm{Si}_{3} \mathrm{~N}_{4}$ ). The diameter $d$ is $50 \mathrm{~nm}, \Lambda$ is varied from 80 to 160 $\mathrm{nm}$, and $\theta=0^{\circ}, 30^{\circ}$, and $60^{\circ}$. The value of $h$ at which intensities are greatest is not strongly dependent on $\Lambda$ for a given $\theta$. Corresponding values of the aspect ratio $(h / d)_{\text {peak }}$ at which intensities are greatest (determined from a polynomial fit of points near the peaks) are plotted versus $\Lambda / d$ in Figure 3 along with results for $\theta=40^{\circ}$. The values of $(h / d)_{\text {peak }}$ are between 0.7 and 1.5. Similar calculated values of $(h / d)_{\text {peak }}$ have been reported by Johnson et al. ${ }^{47}$ for gold nanodisk arrays with $d=$ $30 \mathrm{~nm}$. For higher values of $d,(h / d)_{\text {peak }}$ shifts toward lower values. For example, with $d=70 \mathrm{~nm}, \theta=60^{\circ}$, and $\Lambda=100$ $\mathrm{nm},(h / d)_{\text {peak }}$ for $\left(|\mathbf{E}|^{2} /\left|\mathbf{E}_{0}\right|^{2}\right)_{\text {avg }}$ is $\sim 0.6$.

B. Spacing and Incident Angle. To further explore the dependence of $\left(|\mathbf{E}|^{2} /\left|\mathbf{E}_{0}\right|^{2}\right)_{\text {avg }}$ and $\left(|\mathbf{E}|^{2} /\left|\mathbf{E}_{0}\right|^{2}\right)_{\max }$ on $\Lambda / d$ and $\theta$, we proceed by fixing the value of $h / d$ at 0.8 (within the range of optimal values shown in Figure 3 ). Figure 4 shows plots of $\left(|\mathbf{E}|^{2} /\left|\mathbf{E}_{0}\right|^{2}\right)_{\text {avg }}$ and $\left(|\mathbf{E}|^{2} /\left|\mathbf{E}_{0}\right|^{2}\right)_{\max }$ as a function of $\Lambda / d$ with $\theta$ from $0^{\circ}$ to $70^{\circ}$. For $\theta$ less than $60^{\circ},\left(|\mathbf{E}|^{2} /\left|\mathbf{E}_{0}\right|^{2}\right)_{\text {avg }}$ increases systematically with decreasing $\Lambda / d$ and $\theta$, in a manner similar to that previously reported for arrays of nanodisks with $d=30 \mathrm{~nm} \cdot{ }^{47}$ On the other hand, $\left(|\mathbf{E}|^{2} /\left|\mathbf{E}_{0}\right|^{2}\right)_{\max }$ generally decreases with decreasing $\Lambda / d$ for $\theta$ greater than $10^{\circ}$. Apparent irregularities (nonmonotonic behavior) in the curves for $\left(|\mathbf{E}|^{2} /\left|\mathbf{E}_{0}\right|^{2}\right)_{\max }$ are assumed to arise from the fact that maximum intensities (values at single points) are more sensitive than average intensities to inaccuracies in the calculations associated with the finite dipolegrid spacing in DDSCAT.

C. Field Distributions. Figures 5-7 show representative calculations of field intensities illustrating the trends as a 


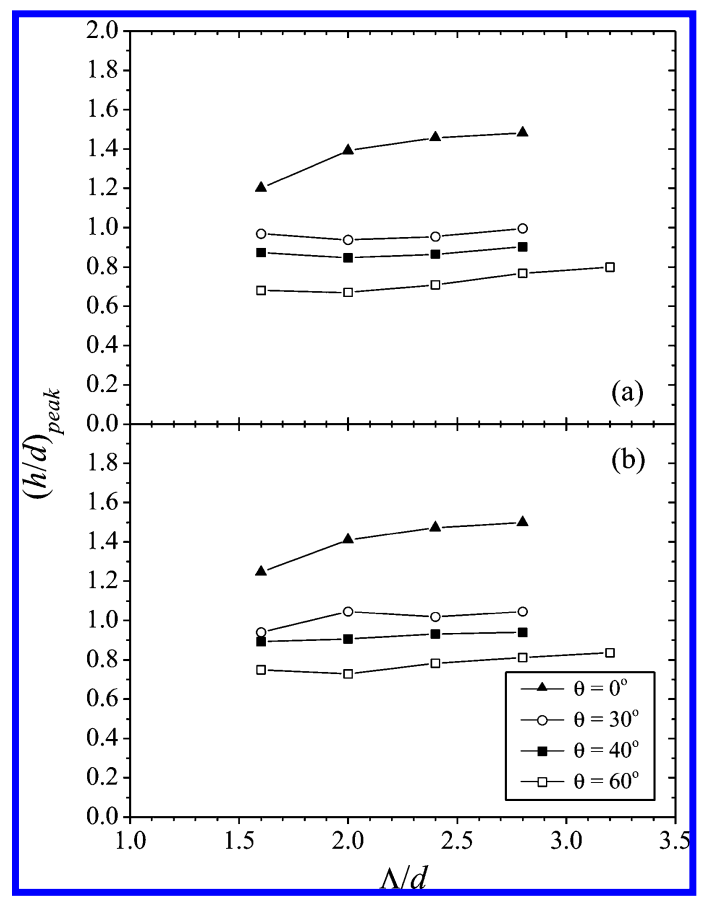

Figure 3. Nanodisk aspect ratio $(h / d)_{\text {peak }}$ that provides the highest (a) $\left(|\mathbf{E}|^{2} /\left|\mathbf{E}_{0}\right|^{2}\right)_{\text {avg }}$ and (b) $\left(|\mathbf{E}|^{2} /\left|\mathbf{E}_{0}\right|^{2}\right)_{\max }$ on surface I for nanodisks with diameter $d=50 \mathrm{~nm}$, plotted as a function of spacing/diameter ratio $\Lambda / d$ for several values of $\theta$. DDSCAT dipole spacing $=5 \mathrm{~nm}$.

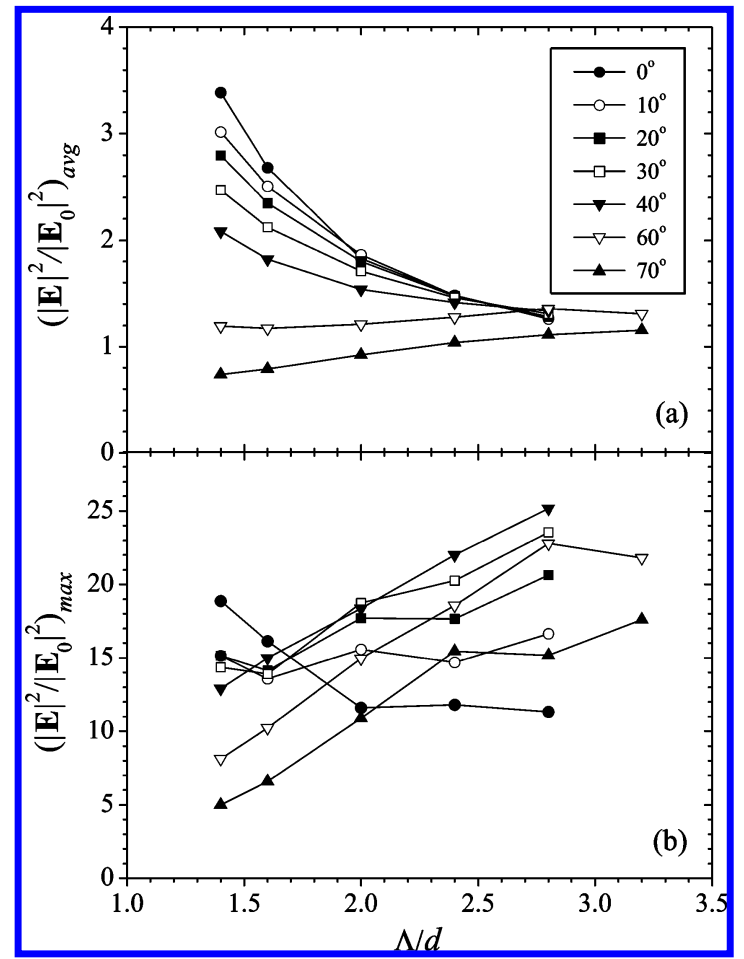

Figure 4. (a) Average intensity $\left(|\mathbf{E}|^{2} /\left|\mathbf{E}_{0}\right|^{2}\right)_{\text {avg }}$ and (b) maximum intensity $\left(|\mathbf{E}|^{2} /\left|\mathbf{E}_{0}\right|^{2}\right)_{\max }$ over surface I as a function of spacing/diameter ratio $\Lambda / d$ with diameter $d=50 \mathrm{~nm}$, height $h=40 \mathrm{~nm}$, and $\theta=0^{\circ}$ to $70^{\circ}$. DDSCAT dipole spacing $=5 \mathrm{~nm}$.

function of $\Lambda$ and $\theta$ for nanodisks with $d=50 \mathrm{~nm}$ and $h=40$ $\mathrm{nm}$. In each of these figures, plot (a) is over the vertical $x-y$ plane $(z=0)$ of the unit cell, and plot (b) is over the horizontal surface I. Slight modulations in the intensities with a periodicity of $2.5 \mathrm{~nm}$ (most apparent on the lower interface of the nanodisk) are calculational artifacts arising from the $2.5 \mathrm{~nm}$ spacing of the discrete dipoles.

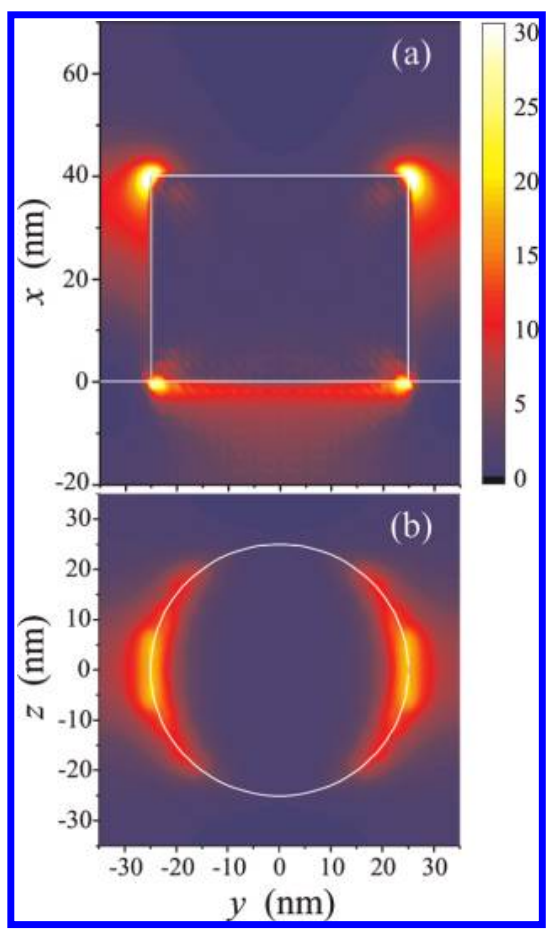

Figure 5. Relative electric-field intensities $|\mathbf{E}|^{2} /\left|\mathbf{E}_{0}\right|^{2}$ over (a) the $x-y$ plane $(z=0)$ and (b) surface I with spacing $\Lambda=70 \mathrm{~nm}, \theta=0^{\circ}$, diameter $d=50 \mathrm{~nm}$, and height $h=40 \mathrm{~nm}$. DDSCAT dipole spacing $=2.5 \mathrm{~nm}$.

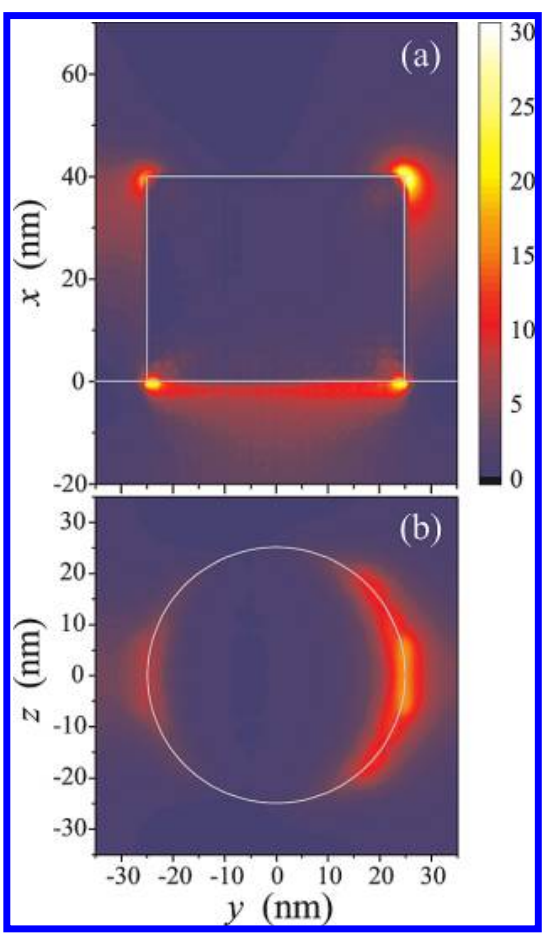

Figure 6. Relative electric-field intensities $|\mathbf{E}|^{2} /\left|\mathbf{E}_{0}\right|^{2}$ over (a) the $x-y$ plane $(z=0)$ and (b) surface I with spacing $\Lambda=70 \mathrm{~nm}, \theta=40^{\circ}$, diameter $d=50 \mathrm{~nm}$, and height $h=40 \mathrm{~nm}$. DDSCAT dipole spacing $=2.5 \mathrm{~nm}$.

The intensity plots in the $x-y$ plane (plot (a) in each of Figures 5-7) show that the greatest intensities in this plane are in the vicinity of the corners of the nanodisks. These highintensity regions extend partially around the top edge of the disk, as shown in the plots over surface I. With $\theta=40^{\circ}$, the intensities on surface I are greatest near the edge of the nanodisk that is more toward the incident light (Figures $6 \mathrm{~b}$ and $7 \mathrm{~b}$ ). As 


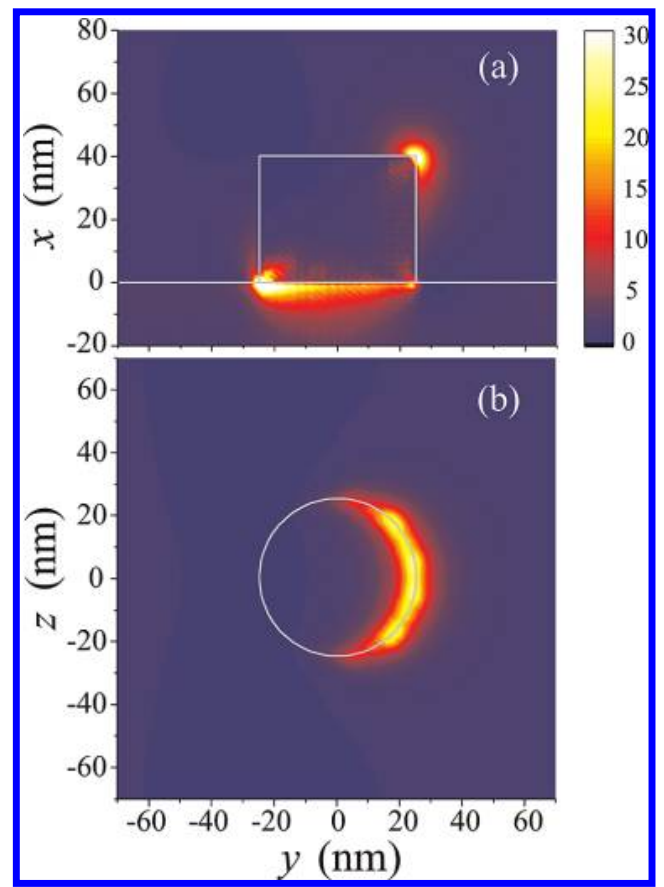

Figure 7. Relative electric-field intensities $|\mathbf{E}|^{2} /\left|\mathbf{E}_{0}\right|^{2}$ over (a) the $x-y$ plane $(z=0)$ and (b) surface I with spacing $\Lambda=140 \mathrm{~nm}, \theta=40^{\circ}$, diameter $d=50 \mathrm{~nm}$, and height $h=40 \mathrm{~nm}$. DDSCAT dipole spacing $=2.5 \mathrm{~nm}$.

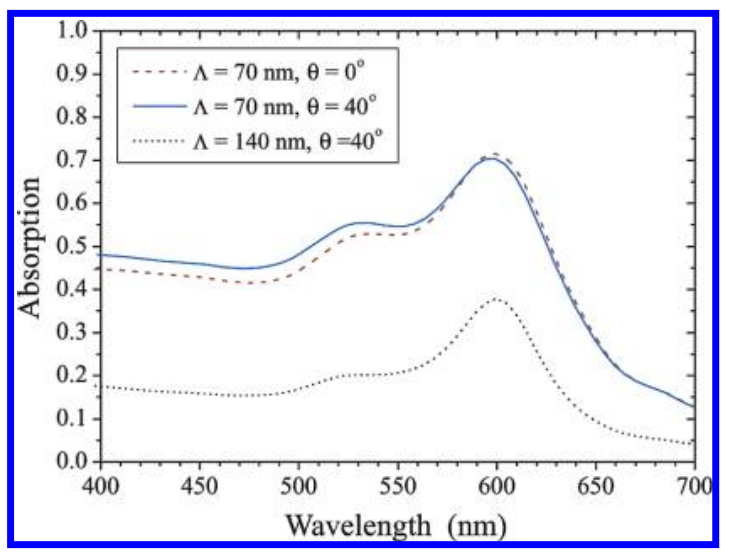

Figure 8. Absorption (fraction of energy not transmitted or scattered) of nanodisk arrays with diameter $d=50 \mathrm{~nm}$ and height $h=40 \mathrm{~nm}$. DDSCAT dipole spacing $=2.5 \mathrm{~nm}$.

either $\Lambda$ or $\theta$ are reduced, intensities near the opposite edge on surface $\mathrm{I}$ increase (Figures $5 \mathrm{~b}$ and $6 \mathrm{~b}$ ).

From the intensity plots, a simple geometric effect can be seen to affect the dependence of $\left(|\mathbf{E}|^{2} /\left.\mathbf{E}_{0}\right|^{2}\right)_{\text {avg }}$ on $\Lambda$. Since the highest intensities on surface I are in regions within a few nanometers of the edges of the nanodisk, $\left(|\mathbf{E}|^{2} /\left|\mathbf{E}_{0}\right|^{2}\right)_{\text {avg }}$ would be approximately inversely proportional to the area of the unit cell, $\Lambda^{2}$, if these intensities remained approximately constant. The increases in $\left(|\mathbf{E}|^{2} /\left.\mathbf{E}_{0}\right|^{2}\right)_{\text {avg }}$ shown in Figure 4 for the lowest angles are consistent with this geometric effect being dominant at these angles.

D. Absorption Spectra. Information on the nature of the surface-plasmon modes that contribute to the fields is provided by the spectra of absorption (fraction of incident energy not transmitted or scattered) plotted in Figure 8 for the same values of $d, \theta$, and $\Lambda$ as those of Figures 5-7. The absorption peak near $600 \mathrm{~nm}$ arises from resonant excitation of the "dipolar" surface-plasmon mode, which has been previously predicted and measured for a variety of gold and silver nanodot array geometries. ${ }^{27-38}$ The field distributions of this mode are not necessarily very similar to a simple dipole because of the lowering of symmetry that arises from the presence of the substrate and nonzero wavevector (when $\theta$ is nonzero). ${ }^{37}$ The peak on the lower-wavelength side of the dipole resonance is understood to arise from a "quadrupolar" surface-plasmon resonance. ${ }^{27,28,32,34-37}$ The maximum of this peak appears in Figure 8 in the range of $520-530 \mathrm{~nm}$, close to the wavelength of $532 \mathrm{~nm}$ that is used in the calculations of Figures 5-7. The broad background of the absorption at wavelengths below the peaks in Figure 8 is understood to arise primarily from interband transitions in the gold, as reflected in the index of refraction. ${ }^{44}$ Consistent with this interpretation, this background is found to not be significantly affected by reducing the imaginary part of the index of refraction $k$ of $\mathrm{Si}_{3} \mathrm{~N}_{4}$ to zero in the calculations (eliminating the contribution of $\mathrm{Si}_{3} \mathrm{~N}_{4}$ to the absorption). For example, at $400 \mathrm{~nm}$, the change in absorption arising from this change in the value of $k$ of $\mathrm{Si}_{3} \mathrm{~N}_{4}$ is less than $3 \%$. The differences in magnitude of the background for the three absorption spectra are associated primarily with differences in the fraction of the unit cell that is gold.

There is no obvious correspondence of changes in the absorption peaks (Figure 8) with changes in $\left(|\mathbf{E}|^{2} /\left|\mathbf{E}_{0}\right|^{2}\right)_{\text {avg }}$ or $\left(|\mathbf{E}|^{2} /\right.$ $\left.\left|\mathbf{E}_{0}\right|^{2}\right)_{\max }$ over surface I as a function of $\Lambda$ and $\theta$ (Figure 4). Detailed analysis of the heights and widths of the peaks is not straightforward, because the damping from interband transitions is strongly dependent on wavelength in the range spanned by the quadrupole and dipole peaks.

Despite the lack of a clear correlation of the absorption with the field intensities, the fact that the maximum absorption occurs at a wavelength substantially above the typical operating wavelengths of Brillouin-scattering systems (515 or $532 \mathrm{~nm}$ ) suggests that field intensities could be increased if the wavelength of the dipole peak were lowered. An approach for achieving this is to use silver, rather than gold, nanodisks or nanolines, since the wavelength of the dipole peak in silver is lower than that of gold. We have explored this option by performing DDSCAT calculations of the absorption of a silver nanodisk array with $d=50 \mathrm{~nm}, \Lambda=70 \mathrm{~nm}, \theta=0^{\circ}$, and $h=$ $40 \mathrm{~nm}$ on a $50 \mathrm{~nm} \mathrm{Si}_{3} \mathrm{Ni}_{4}$ membrane (the same geometric parameters as used for the calculation of one of the curves in Figure 8). This calculation employed values of the indices of refraction reported by Lynch and Hunter. The results show a dipole peak near $502 \mathrm{~nm}$. This indicates that silver nanodisks or nanolines would be more effective in plasmon mediation of BLS, if surface oxidation does not significantly affect the field intensities.

E. Higher-Wavevector Components. As described above, higher-wavevector components of the electric fields on surface I are of particular interest, because they are expected to inelastically scatter from phonons or magnons with correspondingly high wavevectors. In considering these components, it is useful, first, to note that, in the discrete-dipole model for periodic arrays, the polarization $\mathbf{P}_{j l m}$ of a dipole located at

$$
\mathbf{r}_{j l m}=\mathbf{r}_{j 00}+l \mathbf{L}_{y}+m \mathbf{L}_{z}
$$

(where $l$ and $m$ are integers and $\mathbf{L}_{u}$ and $\mathbf{L}_{v}$ are the elementary lattice vectors) is related to the polarization $\mathbf{P}_{j 00}$ of the replica dipole in the unit cell with $l=m=0$ by $^{43}$

$$
\begin{aligned}
\mathbf{P}_{j l m}(t) & =\mathbf{P}_{j 00}(t) \exp \left[i \mathbf{k}_{0} \cdot\left(l \mathbf{L}_{y}+m \mathbf{L}_{z}\right)\right] \\
& =\mathbf{P}_{j 00}(t) \exp \left[i \mathbf{k}_{0} \cdot\left(\mathbf{r}_{j l m}-\mathbf{r}_{j 00}\right)\right]
\end{aligned}
$$


where $j$ is the index for one of the dipoles within a single unit cell. Equation 5 reflects the fact that, for a monochromatic plane wave incident on an infinite periodic structure, the polarizations of equivalent dipoles in two different unit cells must have the same magnitude and phase shift relative to the incident plane wave at their respective locations. The relationship between polarizations in different unit cells can also be expressed as

$$
\mathbf{P}_{j l m}(t)=\mathbf{f}\left(\mathbf{r}_{j l m}\right) \mathrm{e}^{i \omega t} \exp \left(i \mathbf{k}_{0} \cdot\left(\mathbf{r}_{j l m}\right)_{\|}\right)
$$

where $\mathbf{f}\left(\mathbf{r}_{j l m}\right)$ is a function with the periodicity of the array and $\left(\mathbf{r}_{j l m}\right)_{\|}$is the component of $\mathbf{r}_{j l m}$ in the $y-z$ plane. Therefore, the electric field $\mathbf{E}_{\mathrm{d}}$ from the entire ensemble of dipoles has the form

$$
\mathbf{E}_{\mathrm{d}}(t, \mathbf{r})=\mathrm{e}^{i \omega t} \mathbf{F}_{\mathrm{d}}(\mathbf{r}) \exp \left(i \mathbf{k}_{0} \cdot \mathbf{r}_{\|}\right)
$$

where $\mathbf{F}_{\mathrm{d}}(\mathbf{r})$ has the periodicity of the array and $\mathbf{r}_{\|}$is the component of the (continuous) position vector in the $y-z$ plane. The periodicity of $\mathbf{F}_{\mathrm{d}}(\mathbf{r})$ corresponds to the spatial Fourier transform of its dependence on $y$ and $z$ involving only reciprocal lattice vectors $\mathbf{K}$ of the array

$$
\mathbf{E}_{\mathrm{d}}(t, \mathbf{r})=\mathrm{e}^{i \omega t} \sum_{\mathbf{K}} \mathbf{F}_{\mathbf{K}}(x) \exp \left(i \mathbf{K} \cdot \mathbf{r}_{\|}\right) \exp \left(i \mathbf{k}_{0} \cdot \mathbf{r}_{\|}\right)
$$

The total field $\mathbf{E}(t, \mathbf{r})$, which is equal to $\mathbf{E}_{\mathrm{d}}(t, \mathbf{r})+\mathbf{E}_{\text {inc }}(t, \mathbf{r})$, has the same form

$$
\mathbf{E}(t, \mathbf{r})=\mathrm{e}^{i \omega t} \sum_{\mathbf{K}} \mathbf{G}_{\mathbf{K}}(x) \exp \left(i \mathbf{K} \cdot \mathbf{r}_{\|}\right) \exp \left(i \mathbf{k}_{0} \cdot \mathbf{r}_{\|}\right)
$$

with the only difference in Fourier coefficients in this equation and eq 8 being that for $\mathbf{K}=0$.

Expressed in terms of individual field components

$$
\begin{aligned}
& E_{x}(\mathbf{r})=\exp \left(i \mathbf{k}_{0} \cdot \mathbf{r}_{\|}\right) \sum_{\mathbf{K}} G_{\mathbf{K}, x}(x) \exp \left(i \mathbf{K} \cdot \mathbf{r}_{\|}\right) \\
& E_{y}(\mathbf{r})=\exp \left(i \mathbf{k}_{0} \cdot \mathbf{r}_{\|}\right) \sum_{\mathbf{K}} G_{\mathbf{K}, y}(x) \exp \left(i \mathbf{K} \cdot \mathbf{r}_{\|}\right) \\
& E_{z}(\mathbf{r})=\exp \left(i \mathbf{k}_{0} \cdot \mathbf{r}_{\|}\right) \sum_{\mathbf{K}} G_{\mathbf{K}, z}(x) \exp \left(i \mathbf{K} \cdot \mathbf{r}_{\|}\right)
\end{aligned}
$$

where

$$
\begin{gathered}
\mathbf{E}(t, \mathbf{r})=\mathrm{e}^{i \omega t}\left[E_{x}(\mathbf{r}) \hat{x}+E_{y}(\mathbf{r}) \hat{y}+E_{z}(\mathbf{r}) \hat{z}\right] \\
\mathbf{G}_{\mathbf{K}}(\mathbf{r})=G_{\mathbf{K}, x} \hat{x}+G_{\mathbf{K}, y} \hat{y}+G_{\mathbf{K}, z} \hat{z}
\end{gathered}
$$

Therefore, for a given value of $x$, discrete spatial Fourier transforms (DFTs) of exp $\left(-i \mathbf{k}_{0} \cdot \mathbf{r}_{\|}\right)$times each of the field components $E_{x}(\mathbf{r}), E_{y}(\mathbf{r})$, and $E_{y}(\mathbf{r})$ yield the Fourier components $\mathbf{G}_{\mathbf{K}}(x)$.

We are particularly interested in Fourier components at the value of $x$ corresponding to surface I. Table 1 presents results of DFTs of the fields over this surface with $\theta=0$ and $\Lambda=70$
TABLE 1: Sum of Normalized Fourier Coefficients, $\Sigma\left|G_{K}\right|^{2} /\left|E_{0}\right|^{2}$, for Specified $\left|K_{y}\right|$ and $\left|K_{z}\right|$ over Surface $I$ with Spacing $\Lambda=70 \mathrm{~nm}, \theta=0^{\circ}$, Diameter $d=50 \mathrm{~nm}$, and Height $h=40 \mathrm{~nm}$, where $K=K_{y} \hat{y}+K_{z} \hat{z}^{a}$

\begin{tabular}{ccc}
\hline$\left|K_{y}\right| /(2 \pi / \Lambda)$ & $\left|K_{z}\right| /(2 \pi / \Lambda)$ & $\Sigma\left|\mathbf{G}_{\mathbf{K}}\right|^{2} /\left.\mathbf{E}_{0}\right|^{2}$ \\
\hline 0 & 0 & 0.44 \\
1 & 0 & 1.90 \\
2 & 0 & 0.06 \\
0 & 1 & 0.00 \\
0 & 2 & 0.00 \\
1 & 1 & 0.39 \\
2 & 1 & 0.16 \\
1 & 2 & 0.05 \\
2 & 2 & 0.06
\end{tabular}
0.00 .

${ }^{a}$ Calculated values of $\Sigma\left|\mathbf{G}_{\mathbf{K}}\right|^{2} /\left|\mathbf{E}_{0}\right|^{2}$ less than 0.005 are entered as TABLE 2: Sum of Normalized Fourier Coefficients, $\Sigma\left|G_{K}\right|^{2} /\left|E_{0}\right|^{2}$, for Specified $\left|K_{y}\right|$ and $\left|K_{z}\right|$ over Surface I with Spacing $\Lambda=70 \mathrm{~nm}, \boldsymbol{\theta}=\mathbf{4 0}{ }^{\circ}$, Diameter $d=50 \mathrm{~nm}$, and Height $\boldsymbol{h}=40 \mathrm{~nm}$, where $\mathrm{K}=K_{y} \hat{y}+K_{z} \hat{z}$

\begin{tabular}{ccc}
\hline$\left|K_{y}\right| /(2 \pi / \Lambda)$ & $\left|K_{z}\right| /(2 \pi / \Lambda)$ & $\Sigma\left|\mathbf{G}_{\mathbf{K}}\right|^{2} /\left.\mathbf{E}_{0}\right|^{2}$ \\
\hline 0 & 0 & 0.50 \\
1 & 0 & 1.29 \\
2 & 0 & 0.21 \\
0 & 1 & 0.04 \\
0 & 2 & 0.01 \\
1 & 1 & 0.15 \\
2 & 1 & 0.20 \\
1 & 2 & 0.04 \\
2 & 2 & 0.03
\end{tabular}

$\mathrm{nm}$ (the scattering configuration of the total-intensity plot of Figure 5b). Table 2 presents analogous results for $\theta=40^{\circ}$ and $\Lambda=70 \mathrm{~nm}$ (the scattering configuration of the total-intensity plot of Figure 6b). Only results up to $\left|K_{y} /(2 \pi / \Lambda)\right|=\left|K_{z} /(2 \pi / \Lambda)\right|$ $=2$ are presented, where $K_{y}$ and $K_{z}$ are the components of $\mathbf{K}$ along $\hat{y}$ and $\hat{z}$, respectively.

The fields have significant intensities at nonzero values of $K_{y}$ and $K_{z}$. In particular, the intensities of the components of the fields listed in Tables 1 and 2 with $\left|K_{y}\right|$ equal to one elementary reciprocal lattice vector, $2 \pi / \Lambda$, are of the same order of magnitude as that of the incident light. At $\theta=40^{\circ}$, the intensity of this component is somewhat lower than at $\theta=0^{\circ}$, while the intensity of the next-higher-wavevector component, with $\left|K_{y}\right|=4 \pi / \Lambda$, is greater.

The Fourier components summarized in Tables 1 and 2 are made somewhat more complicated by the arrays being periodic in more than one direction. For example, components with $\left|K_{z}\right|$ $=2 \pi / \Lambda$ and $\left|K_{y}\right|=2 \pi / \Lambda$ or $4 \pi / \Lambda$ are significant for both scattering configurations. Any inelastic scattering of these components would involve phonon (or magnon) wavevectors out of the $x-y$ plane. Robertson et al. ${ }^{12}$ describe similar outof-plane interactions for the case of SEBS on Ag gratings. For the purposes of simplifying analysis of SEBS data, it may be preferable to employ arrays of noble-metal nanolines, rather than nanodisks, so that the wavevectors of the incident light, backscattered light, substrate normal, and reciprocal lattice vectors of the array will be in a common plane. Recently, the capabilities of DDSCAT have been extended to enable modeling of nanoline arrays, and initial calculations that we have performed with this code indicate that intensities of the Fourier components in such arrays are comparable in magnitude to those of arrays of nanodisks with similar cross-sectional dimensions and spacings. Since, in these calculations, a $5 \mathrm{~nm}$ layer of $\mathrm{Cr}$ between the substrate and nanolines was included, the results 
also suggest that the use of such a layer is practical for improving adhesion between the $\mathrm{Au}$ and $\mathrm{Si}_{3} \mathrm{~N}_{4}$.

\section{Conclusions}

Average intensities in the plane (surface I) near the top surfaces of the nanodisks are found here to increase with decreasing incident angle $\theta$. At low values of $\theta$, the average intensities in this plane also increase with decreasing array spacing $\Lambda$, and this is attributed primarily to the geometric effect of changing the fractional coverage area of the gold. The optimal aspect ratio $h / d$ of the nanodisks is found to be relatively weakly dependent on $\Lambda$ for a given $\theta$ and to be in the range of 0.7 to 1.5 for $d=50 \mathrm{~nm}$ and the range of $\theta$ studied. These values are similar to those previously reported for arrays of nanodisks with a diameter of $30 \mathrm{~nm}^{47}$

With respect to the feasibility of employing nanodisk or nanoline arrays for plasmon-mediated detection of higherwavevector phonons or magnons in Brillouin scattering, the most important conclusion of this work is represented in Tables 1 and 2. Spatial Fourier components corresponding to the lowest nonzero reciprocal lattice vector along $\hat{y}\left(\left|K_{y}\right|=2 \pi / \Lambda,\left|K_{z}\right|=\right.$ $0)$ are found to have intensities comparable to that of the incident light, and components for other reciprocal lattice vectors are also significant. With $\mathbf{K}=(2 \pi / \Lambda) \hat{y}$ contributing in the wavevector-conservation relation (eq 2), the phonon wavelengths detected in SEBS would be somewhat less than $70 \mathrm{~nm}$ when $\Lambda$ $=70 \mathrm{~nm}$ (being dependent, through eq 2, on the scattering configuation). Considering that state-of-the-art electron-beamlithography systems can achieve resolutions on the order of 10 $\mathrm{nm}$ in thin-film patterning, the results presented here and in ref. ${ }^{47}$ provide evidence for the feasibility of using plasmon mediation of nanodisk or nanoline arrays to extend the range of detectable phonon or magnon wavelengths to a few tens of nanometers or less. These wavelengths are an order of magnitude smaller than those detected in conventional BLS, which have a lower limit of half the wavelength of the incident light.

\section{References and Notes}

(1) Aroca, R. Surface-enhanced vibrational spectroscopv: Wiley: Chichester, 2006.

(2) Ru, E.; Etchegoin, P. Principles of surface-enhanced Raman spectroscopy; Elsevier: Amsterdam, 2008.

(3) Moretti, A. L.; Robertson, W. M.; Fisher, B.; Bray, R. Phvs Rev B 1985, 31 (6), 3361-3368.

(4) Fukui, M.; Toda, O.; So, V. C. Y.; Stegeman, G. I. Solid State Commun. 1980, 36, 995-1000.

(5) Fukui, M.; Tada, O.; So, V. C. Y.; Stegeman, G. I. J. Phys. C: Solid State Phys. 1981, 14, 5591-5607.

(6) Robertson, W. M.; Moretti, A. L.; Bray, R. Phvs. Rev. B 1987, 35 (17), 8919-8928.

(7) Gleed, F. G.; Hillebrands, B.; Lee, S.; Stegeman, G. I.; Sambles, J. R. Solid State Commun. 1989, 70 (3), 237-239.

(8) Eberle, R.; Kresser, T.; Pietralla, M. Thin Solid Films 2002, 408, 169-175, 2002.

(9) Lee, S.; Hillebrands, B.; Dutcher, J. R.; Stegeman, G. I.; Knoll, W.; Nizzoli, F. Phys. Rev. B 1990, 41 (8), 5382-5387.

(10) Eberle, R.; Pietralla, M. Thin Solid Films 2008, 516, 4803-4808.
(11) Robertson, W. M.; Grimsditch, M.; Moretti, A. L.; Kaufman, R. G.; Hulse, G. R.; Fullerton, E.; Schuller, I. K. Phvs. Rev. B 1989, 40, 41534156.

(12) Robertson, W. M.; Grimsditch, M.; Moretti, A. L.; Kaufman, R. G.; Hulse, G. R.; Fullerton, E.; Schuller, I. K. Phys. Rev. B 1990, 41 (8), 49864992.

(13) Utegulov, Z. N.; Shaw, J. M.; Draine, B. T.; Kim, S. A.; Johnson, W. L. Proc. SPIE 2007, 6641, 66411M.

(14) Dutcher, J.; Hillebrands, B.; McLaughlin, G.; Nickel, B.; Stegeman, G. Phvs. Rev. Lett. 1992, 68, 2464.

(15) Lee, S.; Giovanni, L.; Dutcher, J. R.; Nizzoli, F.; Stegeman, G. I.; Marvin, A. M.; Wang, Z.; Ross, J. D.; Amoddeo, A.; Caputi, L. S. Phvs. Rev. B 1994, 49, 2273.

(16) Gorishnyy, T.; Ullal, C.; Maldovan, M.; Fytas, G.; Thomas, E. Phvs. Rev. Lett. 2005, 94, 115501.

(17) Schaadt, D. M.; Feng, B.; Yu, E. T. Appl. Phys. Lett. 2005, 86, 063106.

(18) Pillai, S.; Catchpole, K. R.; Trupke, T.; Green, M. A. J. Appl. Phvs. 2007, 101, 093105.

(19) Lim, S. H.; Mar, W.; Matheu, P.; Derkacs, D.; Yu, E. T. J. Appl. Phvs. 2007, 101, 104309.

(20) Derkacs, D.; Lim, S. H.; Matheu, P. W.; Mar, W.; Yu, E. T. Appl. Phvs. Lett. 2006, 89, 093103.

(21) Hägglund, C.; Zäch, M.; Petersson, G.; Kasemo, B. Appl. Phvs. Lett. 2008, 92, 053110.

(22) Koenderink, A. F.; Hernández, J. V.; Robicheaux, F.; Noordam, L. D.; Polman, A. Nano Lett. 2007, 7, 745.

(23) Kik, P. G.; Martin, A. L.; Maier, S. A.; Atwater, H. A. Proc. SPIE 1998, 4810, 7.

(24) Félidj, N.; Aubard, J.; Levi, G.; Krenn, J. R.; Hohenau, A.; Schider, G.; Leitner, A.; Aussenegg, F. R. Appl. Phvs. Lett. 2003, 82, 3095.

(25) Ozbay, E. Science 2006, 311, 189.

(26) Maier, S. A.; Brongersma, M. L.; Kik, P. G.; Meltzer, S.; Requicha, A. G.; Atwater, H. A. Adv. Mater. 2001, 13 (19), 1501.

(27) Jensen, T. R.; Schatz, G. C.; Van Duyne, R. P. J. Phys. Chem. B 1999, 103, 2394.

(28) Haynes, C. L.; McFarland, A. D.; Zhao, L.; Van Duyne, R. P.; Schatz, G. C. J. Phys. Chem. B 2003, 107, 7337.

(29) Zhao, L.; Kelly, K. L.; Schatz, G. C. J. Phvs. Chem. B 2003, 107, 7343.

(30) Zou, S.; Janel, N.; Schatz, G. C. J. Chem. Phys. 2004, 120, 10871. (31) Chu, T. C.; Tsai, D. P.; Liu, W.-C. J. Kor. Phys. Soc. 2005, 47, S194

(32) Huang, W.; Qian, W.; El-Sayed, M. A. J. Phys. Chem. B 2005, 109, 18881.

(33) Hicks, E. M.; Zou, S. Z.; Schatz, G. C.; Spears, K. G.; Van Duyne, R. P. Nano Lett. 2005, 5 (6), 1065.

(34) Bouhelier, A.; Bachelot, R.; Im, J. S.; Wiederrecht, G. P.; Lerondel, G.; Kostcheev, S.; Royer, P. J. Phvs. Chem. B 2005, 109, 3195.

(35) Pedersen, D. B.; Wang, S. L.; Paige, M. F.; Leontowich, A. F. G. J. Phvs. Chem. C 2007, 111, 5592.

(36) Khlebtsov, B. N.; Khanadeyev, V. A.; Ye, J.; Mackowski, D. W.; Borghs, G.; Khlebtsov, N. G. Phys. Rev. B 2008, 77, 035440.

(37) Gantzounis, G.; Stefanou, N.; Papanikolaou, N. Phvs. Rev. B 2008, 77, 035101.

(38) Zheng, Y. B.; Juluri, B. K.; Mao, X.; Walker, T. R.; Huang, T. J. J. Appl. Phys. 2008, 103, 014308.

(39) Purcell, E. M.; Pennypacker, C. R. Astrophys. J. 1973, 186 (2), 705 .

(40) Draine, B. T.; Goodman, J. Astrophvs. J. 1993, 405 (2), 685.

(41) Draine, B. T.; Flatau, P. J. J. Opt. Soc. Am. A 1994, 11 (4), 1491.

(42) DDSCAT can be downloaded from http://www.astro.princeton.edu/ draine/DDSCAT.html.

(43) Draine, B. T.; Flatau, P. J. J. Opt. Soc. Am. 2008, 25, 2693.

(44) Johnson, P. B.; Christy, R. W. Phvs. Rev. B 1972, 6, 4370.

(45) Philipp, H. R. In Handbook of Optical Constants of Solids; Academic: New York, 1985; p 771.

(46) Djurišić, A. B.; Li, E. H. Appl. Opt. 1998, 37 (22), 5291.

(47) Johnson, W. L.; Kim, S. A.; Utegulov, Z. N.; Draine, B. T. Proc. SPIE 2008, 7032, 70321S.

JP903965D 